05

\title{
Зависимость нагрева стабилизированных нержавеющей сталью токоограничивающих элементов на основе ВТСП-лент второго поколения от воздействия тока и параметров теплоотвода на границе с жидким азотом
}

\author{
(C) С.В. Самойленков ${ }^{1,2}$, В.И. Щербаков ${ }^{2}$, Д.Р. Кумаров ${ }^{3}$, Д.А. Горбунова ${ }^{3}$ \\ ${ }^{1}$ Объединенный институт высоких температур РАН, Москва, Россия \\ 2 ЗАО „СуперОкс“, Москва, Россия \\ ${ }^{3}$ ООО „С-Инновации“, Москва, Россия \\ E-mail: s.samoilenkov@gmail.com
}

Поступило в Редакцию 24 сентября 2019 г.

В окончательной редакции 24 сентября 2019 г.

Принято к публикации 3 октября 2019г.

\begin{abstract}
Экспериментально исследован нагрев током токоограничивающих элементов, изготовленных из ВТСП-лент второго поколения, стабилизированных лентой из нержавеющей стали. Показано, что скорость нагрева мало зависит от степени стабилизации нержавеющей сталью. С использованием математической модели, учитывающей параметры теплоотвода (среда - жидкий азот), продемонстрированы возможности и ограничения увеличения площади токоограничивающего элемента для снижения его температуры в режиме токоограничения.
\end{abstract}

Ключевые слова: токоограничивающие устройства, высокотемпературные проводники, теплоперенос, жидкий азот.

DOI: 10.21883/PJTF.2020.01.48860.18047

Перспективное силовое оборудование для электроэнергетики должно превосходить по техническим характеристикам существующие аналоги: обеспечивать энергосбережение, занимать меньше места, обладать высокой надежностью, быть экологичным и пожаробезопасным. Большинству из этих критериев отвечает электроэнергетическое оборудование на основе высокотемпературных сверхпроводников (ВТСП) [1]. Особенностями ВТСП-устройств являются низкая рабочая температура и использование жидкого азота. Жидкий азот является хорошим диэлектриком и может выполнять функцию электрической изоляции. Нелинейность изменения сопротивления ВТСП при превышении током критической величины позволяет создать токоограничивающее устройство (ВТСП ТОУ). ВТСП ТОУ имеет большие перспективы применения в сетях мегаполисов и промышленных агломераций [2]. Практически все ВТСП ТОУ резистивного типа создаются с применением так называемых ВТСП-проводов второго поколения [3].

Работа токоограничивающих устройств основана на физических свойствах сверхпроводника: ВТСП ТОУ имеет нулевое сопротивление при токе ниже критического и „незаметно“ для сети при работе в номинальном режиме. Ток короткого замыкания вызывает переход ВТСП ТОУ в резистивное состояние, ограничивая тем самым ток. Обеспечение эффективного теплоотвода от проводника в токоограничивающем режиме - ключевая физическая и инженерная задача.
В настоящей работе представлены результаты экспериментальных испытаний токами короткого замыкания токоограничивающих элементов (ТОЭ), изготовленных из ВТСП-провода второго поколения, стабилизированных различным количеством ленты из нержавеющей стали. Полученные экспериментальные результаты сравниваются с расчетами, выполненными на основе модели, описывающей тепловое поведение токопроводящего элемента в токоограничивающем режиме с учетом теплоотвода в жидкий азот.

Токоограничивающие элементы были изготовлены из ВТСП-провода второго поколения российского производства (ООО „С-Инновации“). Средний критический ток ВТСП-провода составил около 600 А на $12 \mathrm{~mm}$ ширины, толщина провода $115 \mu \mathrm{m}$, материал подложки сплав Хастеллой С276, материал сверхпроводника $\mathrm{GdBa}_{2} \mathrm{Cu}_{3} \mathrm{O}_{7}$. ВТСП-провод последовательно покрывался слоем серебра, меди и припоя марки ПОС-61. Общая толщина металлических слоев составляла около $15 \mu \mathrm{m}$. Процесс изготовления ВТСП-провода второго поколения подробно описан в [3]. В токоограничивающем элементе ВТСП-провода соединялись посредством пайки параллельно с лентой из нержавеющей стали. Длина каждого токоограничивающего элемента составляла $50 \mathrm{~cm}$, площадь $800 \mathrm{~cm}^{2}$. К концам токоограничивающего элемента низкотемпературным припоем припаивались массивные медные контакты. Изготовленные элементы отличались различным количеством дополнительных полос, изготовленных из нержавеющей стали марки 
Основные параметры ТОЭ из ВТСП-провода второго поколения в расчете на $1 \mathrm{~m}$ длины

\begin{tabular}{c|c|c|c}
\hline $\begin{array}{c}\text { Номер } \\
\text { ТОЭ }\end{array}$ & $\begin{array}{c}\text { Удельное электрическое } \\
\text { сопротивление при } 293 \mathrm{~K}, \\
\mathrm{~m} \Omega / \mathrm{m}\end{array}$ & $\begin{array}{c}\text { Удельный вес без учета } \\
\text { контактов, } \mathrm{g} / \mathrm{m}\end{array}$ & \begin{tabular}{c} 
Толщина, mm \\
\hline 1
\end{tabular} \\
\hline 17.1 & 160.6 & 0.486 \\
3 & 25.5 & 99.8 & 0.296 \\
& 39.6 & 68.8 & 0.203 \\
\end{tabular}
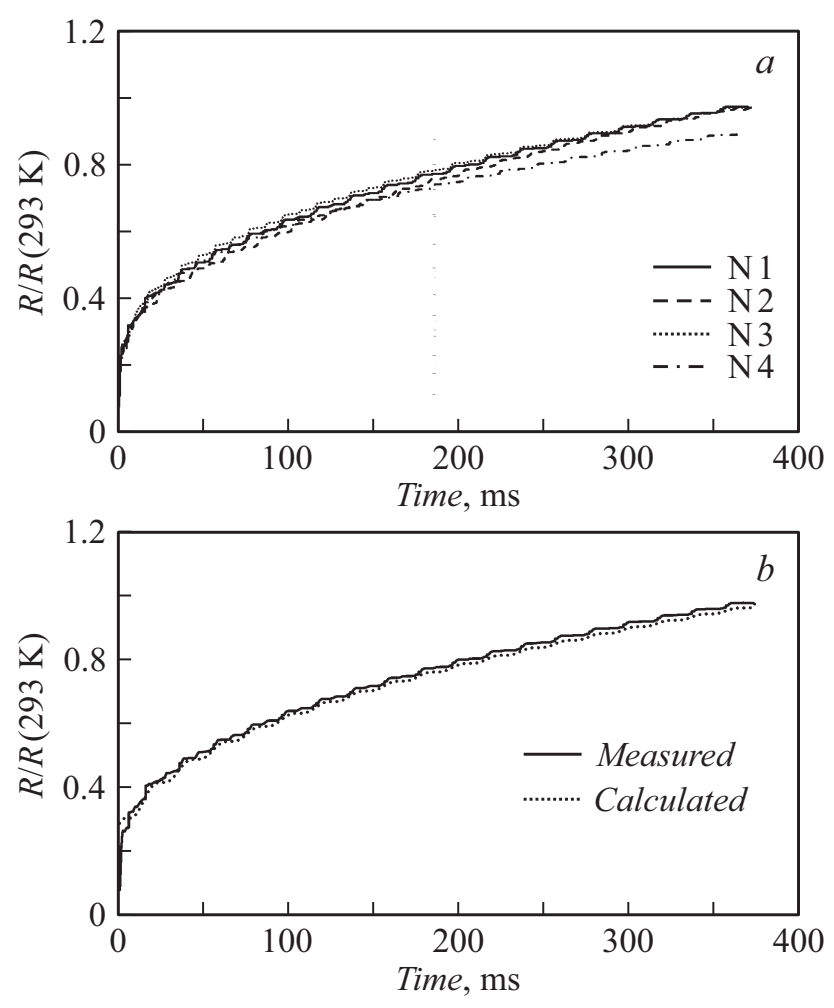

Рис. 1. $a-$ зависимость нормированного на значение при $293 \mathrm{~K}$ сопротивления ТОЭ от времени воздействия напряжения $20 \mathrm{~V} / \mathrm{m} ; b-$ сравнение расчетных и экспериментальных данных для ТОЭ № 1.

ANSI 304, обеспечивающих электрическую, механическую и тепловую стабилизацию элемента (см. таблицу).

Испытания токами короткого замыкания проводились на стенде, включающем в себя понижающий силовой трансформатор, систему цифровой записи значений тока и напряжения. Во время испытаний ВТСП-элементы погружались в жидкий азот, находящийся при атмосферном давлении (температура кипения $77.3 \mathrm{~K}$ ). При помощи ключа в случайный момент времени на образец подавалось переменное напряжение с действующим значением $10 \mathrm{~V}$. Длина импульса напряжения составляла $400 \mathrm{~ms}$. Регистрировались временны́е зависимости напряжения и тока.

Для расчетной модели использовались температурные зависимости теплоемкости [4] и теплоотвода в жидкий азот [5]. Температурные зависимости сопротивления определялись для каждого исследованного ТОЭ экспериментально с помощью четырехконтактного метода.

Математическая модель поведения ТОЭ в режиме короткого замыкания основана на поэтапном расчете характеристик токоограничивающего элемента с малым временны́м шагом $(0.05 \mathrm{~ms})$. Подход состоит в расчете основных параметров ВТСП-провода (выделение и отвод тепла, теплоемкость, электрическое сопротивление, ток) исходя из значения температуры, достигнутого ВТСПпроводом в предыдущей расчетной точке. Допущение модели заключается в том, что за малый период времени изменение перечисленных параметров незначительно и не вносит существенной ошибки в расчеты. Предполагается, что на $1 \mathrm{~m}$ образца действует напряжение с частотой $50 \mathrm{~Hz}$ и значением $20 \mathrm{~V}$. Фаза тока в начальный момент подбиралась для каждого из измеренных образцов индивидуально, так, чтобы максимумы и минимумы на расчетных и экспериментальных кривых совпали.

Результаты экспериментально определенной зависимости сопротивления токоограничивающих элементов от времени показаны на рис. 1,a. Несмотря на существенные различия в конструкции ТОЭ, все они нагреваются при воздействии тока короткого замыкания практически одинаково, достигая комнатной температуры приблизительно за $400 \mathrm{~ms}$ токового импульса. Неравномерность нарастания сопротивления связана с частотой переменного тока, воздействующего на ТОЭ.

При фиксированном напряжении выделяемое джоулево тепло обратно пропорционально сопротивлению, т.е. прямо пропорционально толщине. Теплоемкость тоже прямо пропорциональна толщине элемента. Таким образом, оба фактора нивелируют влияние друг друга.

На рис. $1, b$ экспериментальные данные сравниваются с результатами расчета. Как можно видеть, математическая модель достаточно точно описывает поведение ТОЭ. Максимальное отклонение результатов расчета от эксперимента наблюдается в первые $10 \mathrm{~ms}$, что связано с тем, что модель не учитывает особенности перехода ВТСП-ленты в резистивное состояние. Так, в модели ВТСП-лента считается полностью перешедшей в резистивное состояние в нулевой момент времени, в то время как в эксперименте переход происходит более сложно: сначала происходит переход так называемых 

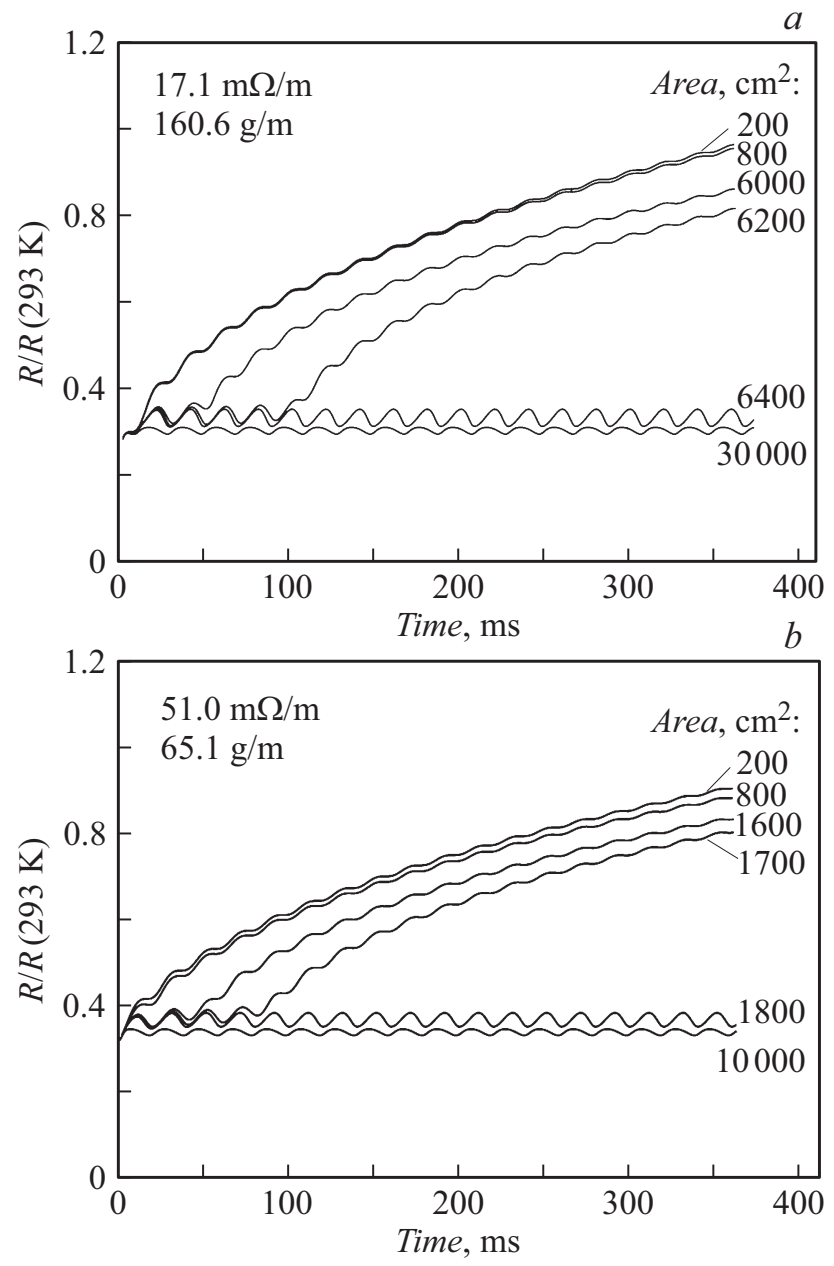

Рис. 2. Расчетные зависимости нормированного сопротивления ТОЭ № $1(a)$ и $4(b)$ при предполагаемом изменении их площади (значения указаны около кривых). Площадь экспериментально исследованных ТОЭ составляла $800 \mathrm{~cm}^{2}$.

„горячих точек“, а резистивное состояние распространяется по ленте с характерной скоростью до $10 \mathrm{~m} / \mathrm{s}$ [6]. В интервале 200-400 ms отклонение расчетной модели от эксперимента составляет менее $2 \%$.

Для практического использования в токоограничивающем устройстве теплоотвод от ТОЭ должен быть максимально эффективным. В случае жидкого азота из-за явления пленочного кипения теплоперенос представляет собой сложную функцию разницы температур между нагретым телом и жидкостью. С другой стороны, площадь токоограничивающего элемента может быть относительно легко изменена, например за счет увеличения его ширины.

Результаты расчета, выполненные для ТОЭ № 1 и 4 с учетом уменьшения или увеличения их площади, приведены на рис. $2, a$ и $b$ соответственно. Для представления на рисунке выбраны значения площади, соответствующие наиболее сильному изменению характера зависимости, а также заведомо низкое и заведомо высокое значения площади. Следует отметить, что уменьшение площади ТОЭ в 4 раза не приводит к заметному изменению зависимостей, что соответствует тому, что нагрев ТОЭ в этих условиях происходит в адиабатическом режиме (теплоприток во много раз превышает теплоотвод).

При увеличении площади ТОЭ и теплоотвода при некотором значении наблюдается скачкообразное изменение характера нагрева. Для более массивного ТОЭ № 1 это значение находится в интервале 6000-6400 $\mathrm{cm}^{2}$, а для ТОЭ № 4 - в интервале $1600-1800 \mathrm{~cm}^{2}$ (рис. 3). В среднем различие составляет 3.6 раза, что больше различия в массе (2.5 раза) и в сопротивлении (3 раза). С учетом зависимости теплоотвода в жидкий азот от температуры охлаждаемого тела [5] это соответствует переходу от низкоэффективного охлаждения к высокоэффективному, которое характерно для разницы температур, не превышающей 20 K. Интересно, что дальнейшее увеличение площади не приводит к существенному изменению температуры. В реальности в области эффективного теплоотвода поведение ТОЭ может сильно отличаться от модели из-за неравномерного по длине ТОЭ нагрева: холодные участки будут охлаждаться более интенсивно, чем горячие, что приведет к дальнейшему росту градиента температуры.

В заключение отметим следующее:

- увеличение стабилизации токоограничивающего ВТСП-элемента нержавеющей сталью не приводит к уменьшению его нагрева при воздействии такого же напряжения;

- разработанная модель позволяет описывать поведение реальных токоограничивающих ВТСП-элементов с точностью около 2\% при времени 200-400 ms;

- температура токоограничивающего ВТСП-элемента в режиме токоограничения может быть значительно снижена, если площадь его будет достаточно большой

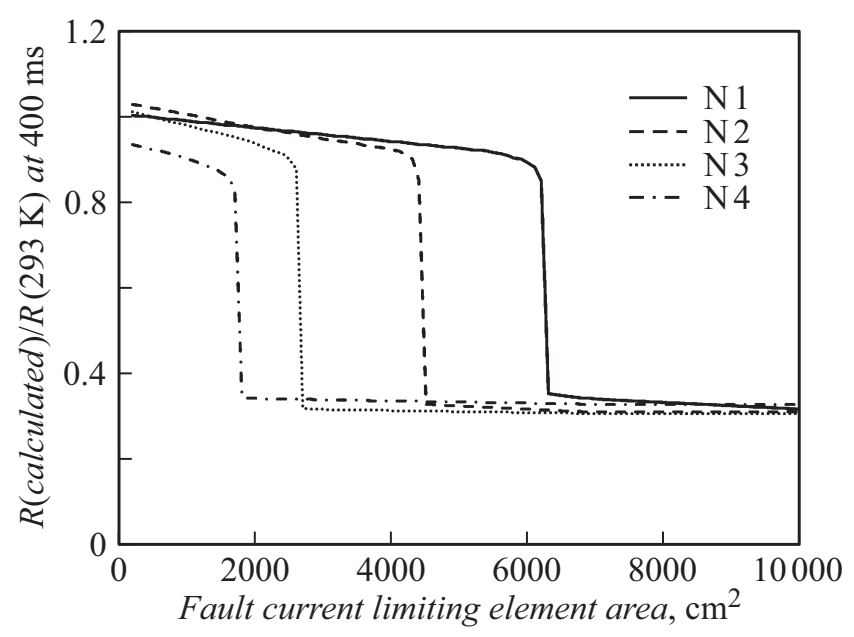

Рис. 3. Рассчитанные значения нормированного на величину при $293 \mathrm{~K}$ сопротивления ТОЭ № 1-4 после воздействия в течение $400 \mathrm{~ms}$ тока при предполагаемом изменении площади элементов. 
для обеспечения эффективного теплоотвода без перехода азота в пленочный режим кипения.

\section{Финансирование работы}

Работа выполнена при поддержке Министерства образования и науки РФ по соглашению № 14.579.21.0141 (уникальный идентификационный номер проекта RFMEFI57917X0141).

\section{Конфликт интересов}

Авторы заявляют, что у них нет конфликта интересов.

\section{Список литературы}

[1] Rey C. Superconductors in the power grid: materials and applications. Woodhead Publ., 2015. 438 p.

[2] Tixador $P$. Superconducting fault current limiter: innovation for the electric grids. World Scientific Publ. Co, 2018. 408 p.

[3] Samoilenkov S., Molodyk A., Lee S., Petrykin V., Kalitka V., Martynova I., Makarevich A., Markelov A., Moyzykh M., Blednov A. // Supercond. Sci. Technol. 2016. V. 29. N 2. P. 024001.

[4] Duthil P. // Materials properties at low temperature. arXiv:1501.07100

[5] Kozak S., Janowski T., Kondratowicz-Kucewicz B., Kozak J., Wojtasiewicz G. // IEEE Trans. Appl. Supercond. 2005. V. 15. N 2. Pt 2. P. 2098-2101.

[6] Antognazza L., Therasse M., Decroux M., Roy F., Dutoit B., Abplanalp M., Fischer O. // IEEE Trans. Appl. Supercond. 2009. V. 19. N 3. Pt 2. P. 1960-1963. 